\title{
SR Pandi-Perumal, Jaime M. Monti and Andrew A. Monjan (eds): Geriatric sleep medicine
}

\author{
Cambridge University Press, Cambridge/UK, 2010, 448 Pages, Price: $95 £$
}

\author{
Nikolaus C. Netzer
}

Received: 28 October 2011 /Revised: 28 October 2011 / Accepted: 8 November 2011 /Published online: 7 December 2011

(C) Springer-Verlag 2011

The hard-covered book within the Sleep Medicine series of the Cambridge University Press by Perumal and colleagues is the first sleep medicine book "concentrating" on sleep in geriatrics. With 41 articles of the same number of author groups and more than 100 authors, it is pretty extensive for this subspecialty. Some authors are famous sleep physicians active in the diverse working groups of the American Academy of Sleep Medicine and American Thoracic Society. Other authors are of non-sleep specialty departments and come from a geriatric perspective of their special field.

Herein lays the problem I see with this book for the nonsleep specialist, general physician and for the sleep specialist. As most books with a collection of articles from different authors, the book contains a large amount of redundancy. Especially the authors not concentrating in their daily work on sleep medicine repeat constantly in the beginning of their articles basic sleep medicine principles for not just the geriatric age group. Reading through the book's chapters, one gets a little bit tired to read about interviewing for general sleep problems over and over again and one tries to find the paragraph where the article gives an answer to specific geriatric sleep questions. The same is true the other way around, most authors write extensively about the general aspects of their specialty, good to see for example on the chapter "Sleep and pain management in the elderly" before they get really to the point of specific geriatric sleep-related problems. On one side, the book gives a lot of information about various medical fields and one, who really reads through the book, gets a lot of information he can use in daily clinical routine. On the other side, I doubt that the book in the shelf will be picked if one searches for a specific answer in pain treatment for example.

A strategic concept for the book with a more extensive editorial work maybe could have avoided the redundancy and some unspecific ties. This would have probably required more articles by the editors counselled by experts in the specific fields. The book of course would have then definitely shrunken in size. Politically, this is not an easy task for editors because they risk the critical comment that they wrote about specialties in which they are not experts. Because the editors Perumal and Monti are pharmacology experts, the book is a little bit too extensive also on the pharmacological treatment side.

So far for the bad news, now some important good aspects with this book: The articles by some of the sleep specialist like Sonia Ancoli Israel are very informative and enlarge the knowledge in geriatric sleep even for experienced sleep specialists, same counts for the Terry Young's chapter about sleep in the menopausal transition and others. It is a big achievement of the book per see that it puts the light on that so far underestimated field of sleep specificities in the growing elderly population. 